\title{
Expression and correlation of Chemerin and FABP4 in peripheral blood of gestational diabetes mellitus patients
}

\author{
XUELING WANG ${ }^{1}$, JUANJUAN LIU ${ }^{2}$, DONGQING WANG ${ }^{3}$, HONGHUA ZHU $^{4}$, LE KANG $^{5}$ and JUAN JIANG ${ }^{6}$ \\ ${ }^{1}$ Department of Obstetrics and Gynecology, The People's Hospital of Zhangqiu Area, Jinan, Shandong 250200; \\ ${ }^{2}$ Department of Obstetrics, The Third People's Hospital of Qingdao, Qingdao, Shandong 266041; \\ Departments of ${ }^{3}$ Obstetrics (Maternal and Child Health Center), ${ }^{4}$ Gastroenterology and \\ ${ }^{5}$ Obstetrics, The People's Hospital of Zhangqiu Area, Jinan, Shandong 250200; \\ ${ }^{6}$ Department of Nursing, The Third People's Hospital of Qingdao, Qingdao, Shandong 266041, P.R. China
}

Received September 20, 2019; Accepted November 18, 2019

DOI: $10.3892 /$ etm.2019.8247

\begin{abstract}
Expression and correlation of Chemerin and fatty acid-binding protein 4 (FABP4) in peripheral blood of gestational diabetes mellitus (GDM) patients were investigated. Sixty patients with GDM from March 2018 to March 2019 in the People's Hospital of Zhangqiu Area were selected as the study group (SG) and another 50 healthy pregnant women corresponding to their age and pregnancy were selected as the control group (CG). Enzyme linked immunosorbent assay (ELISA) was used to detect the expression of Chemerin and FABP4 in serum. Receiver operating characteristic (ROC) curve was used to evaluate the diagnostic value of Chemerin and FABP4 in peripheral blood for GDM patients. Pearson's correlation coefficient was used to analyze the correlation between Chemerin and FABP4 and the correlation between Chemerin and inflammatory factors such as interleukin-6 (IL-6) and tumor necrosis factor- $\alpha$ (TNF- $\alpha$ ). Expression of Chemerin and FABP4 in peripheral blood of GDM patients were significantly higher than those in CG. The AUC of GDM patients diagnosed with Chemerin and FABP4 in peripheral blood was 0.820 and 0.814 , while the AUC of GDM patients diagnosed with Chemerin combined with FABP4 in peripheral blood was 0.904. Expression of inflammatory factors IL-6 and TNF- $\alpha$ in the SG were significantly higher than those in the CG. Chemerin in the SG was positively correlated with FABP4 and positively correlated with inflammatory factors IL-6 and TNF- $\alpha$. Patients with advanced age ( $\geq 35$ years), family history of diabetes, hyperlipidemia, high pre-pregnancy BMI, high fasting blood glucose, high Chemerin and high FABP4 expression have high risk of GDM. In conclusion, Chemerin
\end{abstract}

Correspondence to: Dr Juan Jiang, Department of Nursing, The Third People's Hospital of Qingdao, 29 Yongping Road, Licang, Qingdao, Shandong 266041, P.R. China

E-mail: ejr8d7@163.com

Key words: chemerin, fatty acid-binding protein 4, gestational diabetes mellitus, peripheral blood and FABP4 were upregulated in the peripheral blood of GDM patients. There was a positive correlation between the two and a positive correlation with the inflammatory factors IL-6 and TNF- $\alpha$.

\section{Introduction}

Gestational diabetes mellitus (GDM) is a temporary diabetes mellitus with glucose intolerance during pregnancy. The difference between GDM and diabetes mellitus is sex specificity and temporality, while insulin resistance (IR) is a similar feature between GDM and diabetes mellitus $(1,2)$. According to statistics, GDM accounts for $\sim 2-6 \%$ of the total pregnancies in Europe and nearly half of GDM patients are highly likely to develop diabetes within 10 years (3). In recent years, the methods of early diagnosis and treatment of GDM have been continuously optimized, but early GDM may still lead to poor pregnancy outcomes (4). The pathophysiological mechanism of GDM involves chronic low-grade inflammation, insulin secretion deficiency and abnormal glucose and lipid metabolism caused by obesity (5-7). Therefore, we can explore new early diagnostic tools and potential therapeutic targets for GDM from three angles of improving chronic inflammatory response, increasing insulin sensitivity and maintaining glucose and lipid metabolism balance, which is of great significance for improving pregnancy outcomes of GDM patients.

Chemerin is an inflammatory adipocyte factor and chemoattractant protein secreted by adipocytes. Chemerin has regulatory functions on inflammatory state, fat formation and glycolipid homeostasis and is closely related to IR (8-10). Studies have shown that high levels of Chemerin are associated with poor prognosis of preeclampsia, polycystic ovary syndrome, GDM and other pregnancy diseases (11-13). In the studies of Yang et al (14), serum Chemerin level was upregulated in GDM patients. The higher the Chemerin level of GDM patients in early pregnancy, the greater the risk of GDM development. It suggested that Chemerin can be used as a predictive marker of GDM development risk. The fatty acid-binding protein (FABP) family is a group of small molecular proteins that act as fatty acid transporters in cells, while FABP4 
plays a key role in lipid metabolism as a member of FABP family (15). Studies have shown that FABP4 can enhance insulin sensitivity and reduce atherosclerosis. Knockdown of FABP4 gene can reduce the expression of inflammation-driven macrophage receptor (16). Ning et al (17), reported that FABP4 was overexpressed in GDM patients and has a significant positive correlation with IR and inflammatory factor TNF- $\alpha$, suggesting that FABP4 can be used as a new biomarker for GDM. Chemerin and FABP4 are both inflammatory adipocyte factors expressed in adipocytes, both of which are related to the development and progression of GDM (18).

At present, there are few reports on the expression and correlation of Chemerin and FABP4 in the peripheral blood of GDM patients. Therefore, we explored the diagnostic value and potential therapeutic methods of Chemerin and FABP4 in GDM patients by detecting the expression of Chemerin and FABP4 in the peripheral blood of GDM patients.

\section{Patients and methods}

Baseline data. Sixty patients with GDM admitted to the People's Hospital of Zhangqiu Area (Jinan, China) from March 2018 to March 2019 were selected as the SG and another 50 healthy pregnant women corresponding in age and pregnancy were selected as the CG. In the SG, the age was 20-35 years with an average age of $27.33 \pm 4.75$ years. In the $C G$, the age was 20-35 years with an average age of 27.80 \pm 5.27 years. The study was approved by the Ethics Committee of the People's Hospital of Zhangqiu Area. The subjects and family members signed an informed consent form.

Inclusion and exclusion criteria. Inclusion criteria: Patients who met the standards formulated by the American Diabetes Association (19) in 2012. After $75 \mathrm{~g}$ glucose tolerance test at 24-28 weeks of gestation, GDM was diagnosed if any one of following was present: fasting blood glucose over $5.1 \mathrm{mmol} / \mathrm{l}$, blood glucose over $10.0 \mathrm{mmol} / \mathrm{l}$ for $1 \mathrm{~h}$, blood glucose over $8.5 \mathrm{mmol} / \mathrm{l}$ for $2 \mathrm{~h}$. The age range was $20-35$ years. The patient was informed and agreed to cooperate with the study.

Exclusion criteria: Patients with communication barrier or severe mental disorder; patients comorbid with malignant tumor or serious cardiac, lung, liver, kidney and other dysfunction; pregnant women; patients with hypertension or endocrine and metabolic diseases before pregnancy.

Detection methods. Elbow venous blood $(5 \mathrm{ml})$ was extracted from subjects on an empty stomach in the morning and then placed in a vacuum tube without anticoagulant and centrifuged at $2,600 \mathrm{x} \mathrm{g}$ for $10 \mathrm{~min}$ at $4^{\circ} \mathrm{C}$. The serum was stored in EP tube for later use and placed in a low temperature refrigerator at $-75^{\circ} \mathrm{C}$. The serum was taken from the freezer, placed it in a refrigerator at $4^{\circ} \mathrm{C}$ for dissolution, and then placed it at room temperature for complete dissolution. Enzyme linked immunosorbent assay (ELISA) (20) was used to detect the expression of Chemerin, FABP4, interleukin-6 (IL-6) and tumor necrosis factor- $\alpha(\mathrm{TNF}-\alpha)$ in serum. The tests were carried out in strict accordance with the specifications of human Chemerin ELISA kit, human FABP4 ELISA kit, human IL-6 ELISA kit and human TNF- $\alpha$ ELISA kit (Shanghai Zhenyu Biotechnology Co., Ltd.; CSB-E10398h, CSB-E12995h, E-EL-H0102km,
E-EL-H0109km). The sample well, standard sample well and blank well were set up. Sample $(50 \mu \mathrm{l})$ to be tested was added to the sample well. The standard sample (50 $\mu \mathrm{l})$ was added to the standard sample well. No reagent was added to blank well. Horseradish peroxidase labeled detection antibody $(100 \mu \mathrm{l})$ was added to the sample well and the standard sample well, then the plate was sealed and incubated at $37^{\circ} \mathrm{C}$ for $60 \mathrm{~min}$. The liquid was discarded, shaken off and repeatedly washed 5 times. The substrates A and B were fully mixed to volume of 1:1. Then $(100 \mu \mathrm{l})$ of substrate mixed solution was added to each well. The plates were sealed and incubated at $37^{\circ} \mathrm{C}$ for $15 \mathrm{~min}$. Terminal liquid $(50 \mu \mathrm{l})$ was added to each well. The absorbance (OD value) at $450 \mathrm{~nm}$ of each well was read by a fully-automatic enzyme-labeled analyzer (M15; Shanghai Chenlian Biotechnology Development Co., Ltd.). The expression of Chemerin, FABP4, IL- 6 and TNF- $\alpha$ were calculated.

Statistical analysis. SPSS 19.0 (IBM Corp.) statistical data software was used for statistical analysis. GraphPad Prism6 (GraphPad Software) was used to draw the data. Enumeration data was expressed by the number of samples/percentage [n $(\%)]$. The Chi-square test was used for comparison of enumeration data between groups. The measurement data were expressed as mean number \pm standard deviation (mean $\pm \mathrm{SD}$ ). The independent-sample t-test was used to compare the measurement data between groups. Receiver operating characteristic (ROC) curve was used to evaluate the diagnostic value of peripheral blood Chemerin and FABP4 in GDM patients. Pearson's correlation coefficient was used to analyze the correlation between Chemerin and FABP4 as well as the correlation with inflammatory factors IL-6 and TNF- $\alpha$. Logistic multivariate regression analysis was used to analyze the independent risk factors affecting GDN. $\mathrm{P}<0.05$ was considered statistically significant.

\section{Results}

Baseline data. There was no significant difference between the two groups in baseline data of height, gestational age, abdominal circumference, systolic blood pressure, diastolic blood pressure, postprandial insulin for $0.5 \mathrm{~h}$, postprandial insulin for $1 \mathrm{~h}$, postprandial insulin for $2 \mathrm{~h}$, total cholesterol ( $\mathrm{P}>0.05)$, but there was a significant difference in baseline data of age, diabetes history, hyperlipidemia, pre-pregnancy $\mathrm{BMI}$, increase of body mass during pregnancy, fasting blood glucose, fasting insulin, IR index (HOMA-IR), Chemerin or FABP4 $(\mathrm{P}<0.05)$ (Table I).

Expression of Chemerin and FABP4 of patients in the two groups. Expression of Chemerin was 5.78 \pm 1.35 and $7.71 \pm 2.23 \mu \mathrm{g} / 1$ in $\mathrm{CG}$ and $\mathrm{SG}$, respectively, while expression of FABP4 was $21.53 \pm 8.89$ and $35.14 \pm 11.39 \mu \mathrm{g} / \mathrm{l}$ in CG and SG, respectively. Expression of Chemerin in CG was significantly lower than that in $\mathrm{SG}(\mathrm{P}<0.001)$. The expression of FABP4 in CG was significantly lower than that in SG $(\mathrm{P}<0.001)$ (Fig. 1).

Diagnostic value of Chemerin and FABP4 in GDM patients. The ROC curve of peripheral blood of Chemerin in the diagnosis of GDM patients was plotted and it was found that the AUC of peripheral blood of Chemerin in the diagnosis of GDM patients was 0.820 (95\% CI, 0.744-0.896), the cut-off 
Table I. Comparison of baseline data of patients between the two groups [n(\%), mean \pm SD].

\begin{tabular}{|c|c|c|c|c|}
\hline Category & $\mathrm{CG}(\mathrm{n}=50)$ & $\mathrm{SG}(\mathrm{n}=60)$ & $\chi^{2} / \mathrm{t}$ value & P-value \\
\hline Age/years & & & 3.976 & 0.046 \\
\hline$\geq 35$ & $7(14.00)$ & $18(28.33)$ & & \\
\hline$<35$ & $43(86.00)$ & $42(71.67)$ & & \\
\hline Diabetes history & & & 13.943 & 0.002 \\
\hline Yes & $8(16.00)$ & $30(50.00)$ & & \\
\hline No & $42(84.00)$ & $30(50.00)$ & & \\
\hline Hyperlipidemia & & & 4.125 & 0.042 \\
\hline Yes & $5(10.00)$ & $15(25.00)$ & & \\
\hline No & $45(90.00)$ & $45(75.00)$ & & \\
\hline Height $(\mathrm{cm})$ & $161.54 \pm 5.23$ & $162.01 \pm 5.12$ & 0.637 & 0.526 \\
\hline Pre-pregnancy BMI (kg/m²) & & & 4.073 & 0.044 \\
\hline$\geq 23$ & $11(26.00)$ & $24(35.00)$ & & \\
\hline$<23$ & $39(74.00)$ & $36(65.00)$ & & \\
\hline Increase of body mass during pregnancy (kg) & $13.52 \pm 4.26$ & $15.61 \pm 4.53$ & 2.475 & 0.015 \\
\hline Gestational age (week) & $23.85 \pm 1.85$ & $24.45 \pm 1.55$ & 1.851 & 0.067 \\
\hline Abdominal circumference $(\mathrm{cm})$ & $99.98 \pm 6.36$ & $101.87 \pm 6.65$ & 1.514 & 0.133 \\
\hline Systolic blood pressure (mmHg) & $114.12 \pm 9.06$ & $115.59 \pm 8.99$ & 0.851 & 0.397 \\
\hline Diastolic blood pressure (mmHg) & $72.98 \pm 7.16$ & $75.04 \pm 6.88$ & 1.535 & 0.128 \\
\hline Fasting blood glucose (mmol/l) & $4.58 \pm 0.35$ & $6.13 \pm 0.89$ & 11.580 & $<0.001$ \\
\hline Fasting insulin $(\mathrm{mU} / \mathrm{l})$ & $9.12 \pm 4.67$ & $13.19 \pm 5.15$ & 4.304 & $<0.001$ \\
\hline Postprandial insulin for $0.5 \mathrm{~h}(\mathrm{mU} / \mathrm{l})$ & $71.39 \pm 37.85$ & $67.88 \pm 24.05$ & 0.590 & 0.557 \\
\hline Postprandial insulin for $1 \mathrm{~h}(\mathrm{mU} / \mathrm{l})$ & $90.87 \pm 34.58$ & $83.81 \pm 23.66$ & 1.266 & 0.208 \\
\hline Postprandial insulin for $2 \mathrm{~h}(\mathrm{mU} / \mathrm{l})$ & $70.27 \pm 22.64$ & $80.85 \pm 36.09$ & 1.798 & 0.075 \\
\hline HOMA-IR & $6.42 \pm 1.81$ & $22.35 \pm 13.88$ & 8.053 & $<0.001$ \\
\hline Total cholesterol (mmol/l) & $5.92 \pm 1.43$ & $6.03 \pm 1.28$ & 0.426 & 0.671 \\
\hline Chemerin $(\mu \mathrm{g} / \mathrm{l})$ & $5.78 \pm 1.35$ & $7.71 \pm 2.23$ & 5.354 & $<0.001$ \\
\hline $\mathrm{FABP} 4(\mu \mathrm{g} / \mathrm{l})$ & $21.53 \pm 8.89$ & $35.14 \pm 11.39$ & 6.880 & $<0.001$ \\
\hline
\end{tabular}

FABP4, fatty acid-binding protein 4; SG, study group; CG, control group.

Table II. ROC parameters of Chemerin and FABP4 in diagnosis of GDM patients.

\begin{tabular}{lcccrrr}
\hline Grouping & AUC & $95 \%$ CI & SE & Cut-off & Sensitivity (\%) & Specificity (\%) \\
\hline Chemerin & 0.820 & $0.744-0.896$ & 0.039 & 6.78 & 73.33 & 76.00 \\
FABP4 & 0.814 & $0.733-0.895$ & 0.041 & 27.64 & 75.00 & 80.00 \\
Chemerin + FABP4 & 0.904 & $0.837-0.952$ & 0.029 & 0.71 & 80.00 & 96.00 \\
\hline
\end{tabular}

FABP4, fatty acid-binding protein 4; GDM, gestational diabetes mellitus; ROC, receiver operating characteristic.

value was 6.78 , the sensitivity was $73.33 \%$ and the specificity was $76.00 \%$. AUC of peripheral blood FABP4 in the diagnosis of GDM patients was 0.814 (95\% CI, 0.733-0.895), the cut-off value was 27.64 , the sensitivity was $75.00 \%$ and the specificity was $80.00 \%$. Then, the two single factors, Chemerin and FABP4, were used as independent variables to conduct binomial Logistic regression analysis. Logistic regression model was obtained: $\operatorname{Logit}(\mathrm{p})=-8.73+1.663$ chemerin +27.574
FABP4. The AUC of the model for diagnosis of GDM patients was 0.904 (95\% CI, 0.837-0.952), the cut-off value was 0.71 , the sensitivity was $80.00 \%$ and the specificity was $96.00 \%$ (Fig. 2 and Table II).

Expression of inflammatory factors IL- 6 and TNF- $\alpha$ of patients in the two groups and the correlation with Chemerin and FABP4. The expression of inflammatory factor IL-6 in 
A

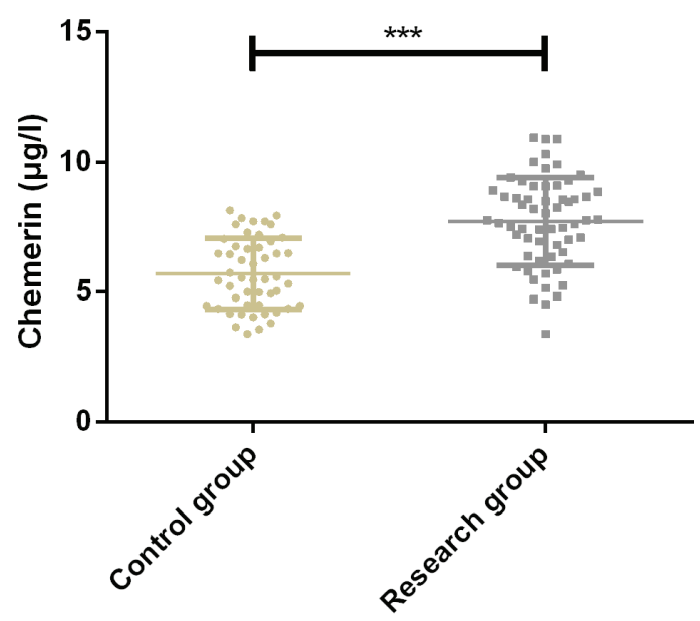

B

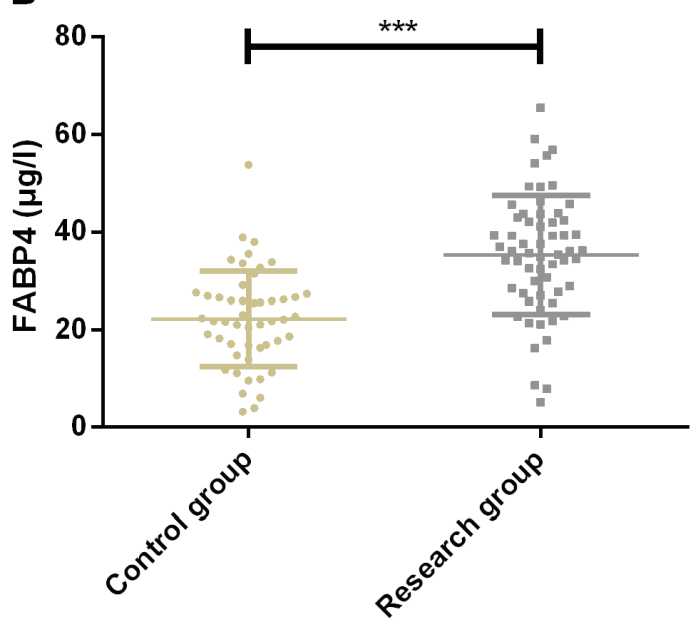

Figure 1. Expression results of Chemerin and FABP4 of patients in the two groups. (A) The expression of Chemorin in SG was significantly higher than that in CG. (B) The expression of FABP4 in SG was significantly higher than that in $\mathrm{CG}$. ${ }^{* * *} \mathrm{P}<0.001$ compared with the control group. FABP4, fatty acid-binding protein 4; SG, study group; CG, control group.

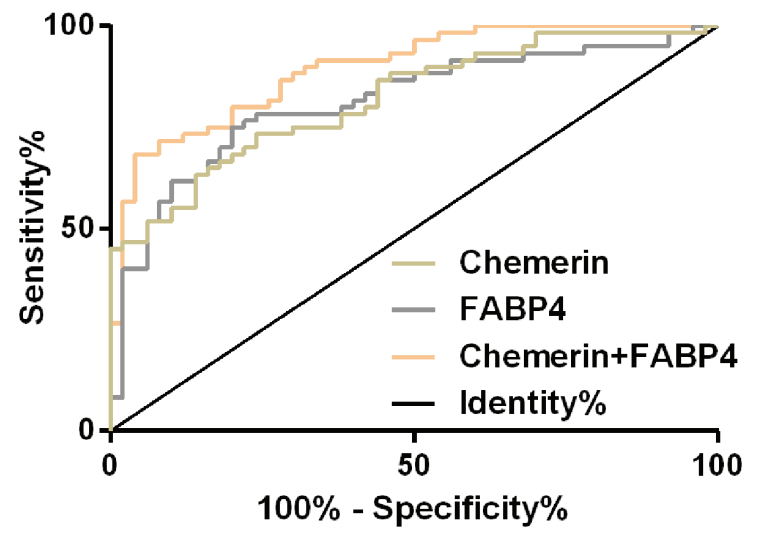

Figure 2. ROC curve of Chemerin and FABP4 in diagnosis of GDM patients. ROC, receiver operating characteristic; FABP4, fatty acid-binding protein 4; GDM, gestational diabetes mellitus.

CG and SG was $83.89 \pm 5.74$ and $98.34 \pm 8.98$, respectively. The expression of inflammatory factor TNF- $\alpha$ in CG and SG were $84.58 \pm 7.38$ and $130.24 \pm 12.02$, respectively. The expression of inflammatory factors IL-6 and TNF- $\alpha$ in CG was significantly lower than that in SG $(\mathrm{P}<0.001)$. Pearson correlation coefficient was used to analyze the correlation between chemerin, FABP4 and inflammatory factors IL-6, TNF- $\alpha$. The results showed that chemerin and FABP4 were positively correlated with inflammatory factors IL-6 and TNF- $\alpha(\mathrm{r}=0.658, \mathrm{P}<0.001 ; \mathrm{r}=0.672, \mathrm{P}<0.001 ; \mathrm{r}=0.648$, $\mathrm{P}<0.001 ; \mathrm{r}=0.649, \mathrm{P}<0.001$ ) (Fig. 3).

Multiple Logistic regression analysis of GDM. Multivariate Logistic regression analysis was conducted on the factors with differences. The results showed that age $(\mathrm{P}=0.002)$, diabetes history $(\mathrm{P}=0.007)$, hyperlipidemia $(\mathrm{P}=0.021)$, pre-pregnancy BMI $(\mathrm{P}=0.010)$, fasting blood glucose $(\mathrm{P}=0.002)$, Chemerin $(\mathrm{P}=0.004)$ and FABP4 $(\mathrm{P}=0.001)$ were independent risk factors for affecting GDM. Patients with advanced age ( $\geq 35$ years), family history of diabetes, hyperlipidemia, high pre-pregnancy BMI, high fasting blood glucose, high
Table III. Assignment of logistic multivariate regression analysis.

\begin{tabular}{lcc}
\hline Factors & Variables & Assignment \\
\hline Age & X1 & $<35=0, \geq 35=1$ \\
Family history of diabetes & X2 & No=0; Yes $=1$ \\
Hyperlipidemia & X3 & No=0; Yes $=1$ \\
Pre-pregnancy BMI & X4 & Continuous variables \\
Increase of body mass & X5 & Continuous variables \\
during pregnancy & & \\
Fasting blood glucose & X6 & Continuous variables \\
Fasting insulin & X7 & Continuous variables \\
HOMA-IR & X8 & Continuous variables \\
Chemerin & X9 & Continuous variables \\
FABP4 & X10 & Continuous variables \\
\end{tabular}

FABP4, fatty acid-binding protein 4 .

Chemerin and high FABP4 expression have increased risk of GDM (Tables III and IV).

Correlation analysis of Chemerin and FABP4. The correlation between Chemerin and FABP4 was analyzed by Pearson's correlation coefficient. The results showed that peripheral blood of Chemerin was positively correlated with FABP4 in $\mathrm{SG}(\mathrm{r}=0.712$, $\mathrm{P}<0.001)$ (Fig. 4).

\section{Discussion}

GDM is a heterogeneous multivariate pregnancy disease with complicated pathological mechanism. In addition to inflammatory reaction, IR and abnormality of lipid and glucose metabolism, GDM also involves DNA methylation and oxidative stress signal transduction that affect cardiac function (21). Statistics showed that the increase in the prevalence of GDM is global and $33.33 \%$ of GDM pregnant women will suffer from 
A

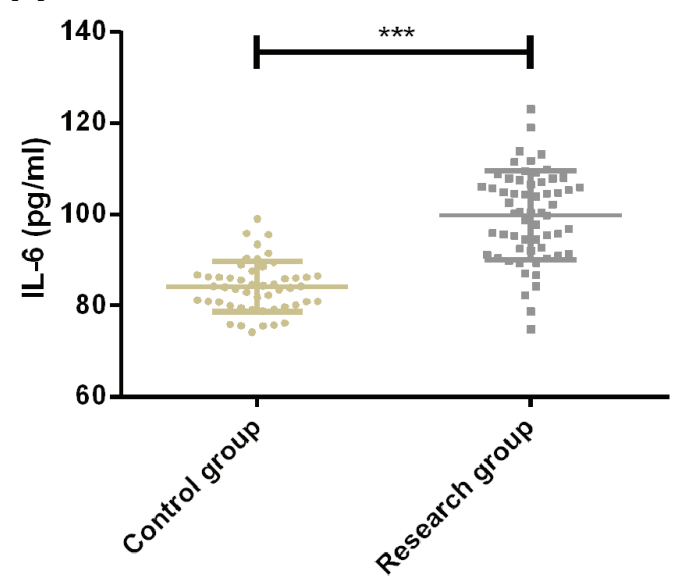

C

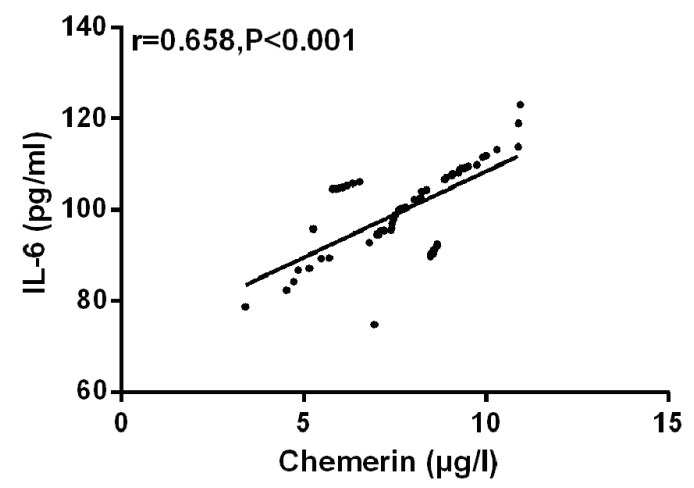

E

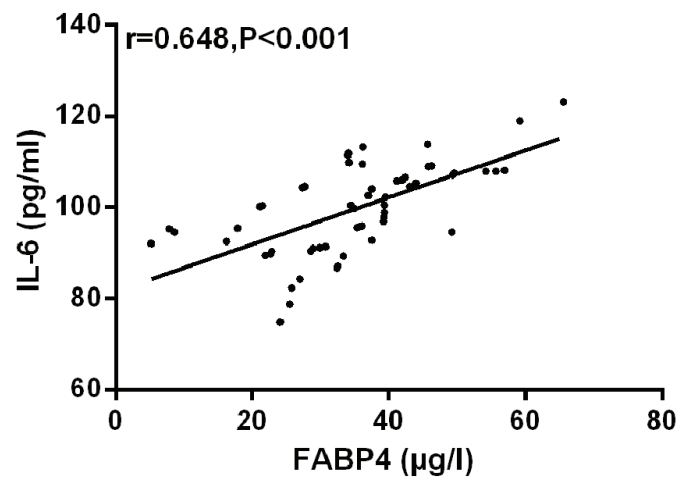

B

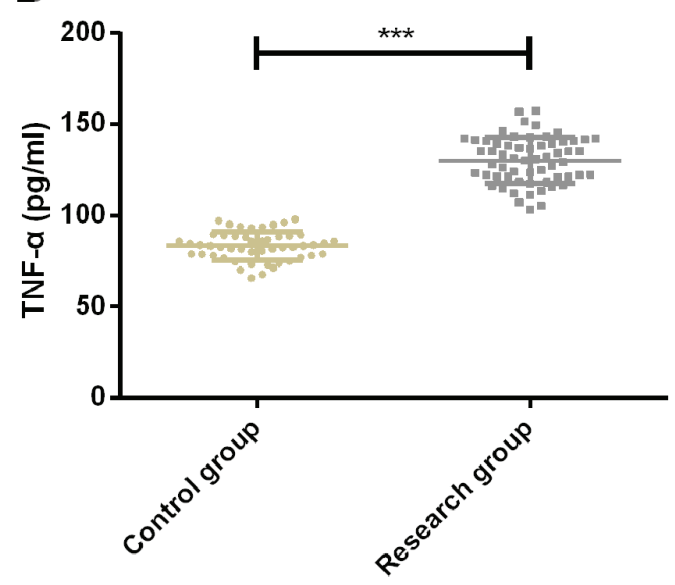

D

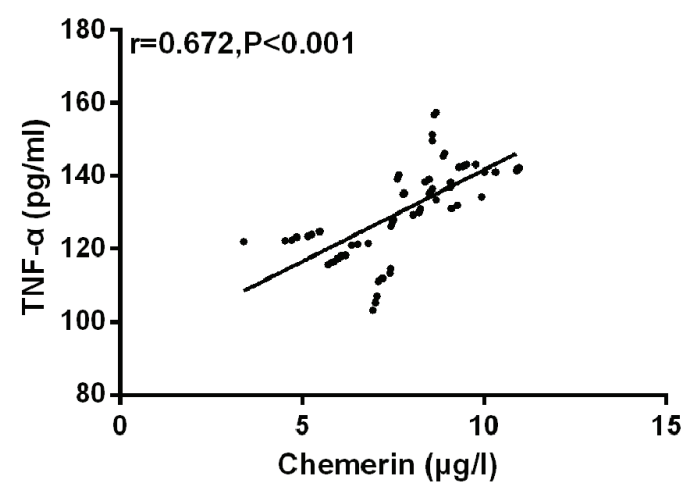

$\mathbf{F}$

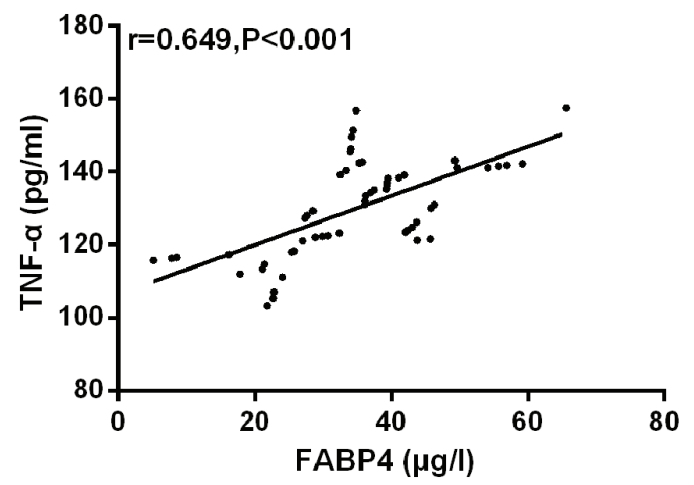

Figure 3. Expression of IL-6 and TNF- $\alpha$ of patients in two groups and their correlation results with Chemerin and FABP4. (A) The expression of inflammatory factor IL-6 in SG was significantly higher than that in CG. (B) The expression of inflammatory factor TNF- $\alpha$ in SG was significantly higher than that in CG. (C) Chemerin was positively correlated with inflammatory factor IL-6 ( $\mathrm{r}=0.658, \mathrm{P}<0.001)$. (D) Chemerin was positively correlated with inflammatory factor TNF- $\alpha(\mathrm{r}=0.672, \mathrm{P}<0.001)$. (E) FABP4 was positively correlated with inflammatory factor IL-6 ( $\mathrm{r}=0.648, \mathrm{P}<0.001)$. (F) FABP4 was positively correlated with inflammatory factor TNF- $\alpha(\mathrm{r}=0.649, \mathrm{P}<0.001) .{ }^{* * *} \mathrm{P}<0.001$ compared with the control group. FABP4, fatty acid-binding protein 4; SG, study group; CG, control group; IL-6, interleukin-6; TNF- $\alpha$, tumor necrosis factor- $\alpha$.

postpartum depression $(22,23)$. In order to avoid the possible serious impact of GDM on the health of pregnant women and newborns, we advocate healthy diet and reasonable physical exercise for women during pregnancy. Some studies have shown that this has preventive effect on GDM (24).

In this study, the expression of peripheral blood of Chemerin and FABP4 in GDM patients was significantly upregulated compared with the CG. The AUC of peripheral blood of Chemerin and FABP4 for diagnosis of GDM patients was 0.820 and 0.814, while the AUC of peripheral blood of Chemerin combined with FABP4 for diagnosis of GDM patients was 0.904, indicating that peripheral blood of Chemerin combined with FABP4 has excellent diagnostic value for diagnosis of GDM patients and can be used as biomarker for prediction of GDM. In the study of Francis et al (25) on adipocyte factors and GDM risks, the concentrations of chemerin and FABP4 in GDM patients were significantly higher than those in CG and both were significantly positively correlated with GDM risks, 
Table IV. Multiple Logistic regression analysis of GDM.

\begin{tabular}{|c|c|c|c|c|c|c|}
\hline Variables & $\mathrm{B}$ & SE & Wals & P-value & OR & $95 \% \mathrm{CI}$ \\
\hline Age & 0.143 & 0.048 & 9.394 & 0.002 & 1.153 & $1.054-1.619$ \\
\hline Family history of diabetes & 0.579 & 0.192 & 7.040 & 0.007 & 1.784 & $1.165-2.751$ \\
\hline Hyperlipidemia & 0.174 & 0.080 & 5.001 & 0.021 & 1.897 & $1.003-1.343$ \\
\hline Pre-pregnancy BMI & 0.742 & 0.305 & 6.622 & 0.010 & 2.118 & $1.189-3.734$ \\
\hline Increase of body mass during pregnancy & 0.101 & 0.473 & 0.045 & 0.829 & 1.106 & $0.429-2.819$ \\
\hline Fasting blood glucose & 0.338 & 0.108 & 9.935 & 0.002 & 1.399 & $1.137-1.736$ \\
\hline Fasting insulin & 0.634 & 0.599 & 1.145 & 0.280 & 1.901 & $0.571-6.152$ \\
\hline HOMA-IR & 0.945 & 0.703 & 1.732 & 0.179 & 2.563 & $0.624-10.105$ \\
\hline Chemerin & 1.345 & 0.475 & 8.617 & 0.004 & 4.029 & $1.598-10.217$ \\
\hline FABP4 & 1.612 & 0.471 & 11.658 & 0.001 & 5.005 & $1.942-12.618$ \\
\hline
\end{tabular}

FABP4, fatty acid-binding protein 4; GDM, gestational diabetes mellitus.

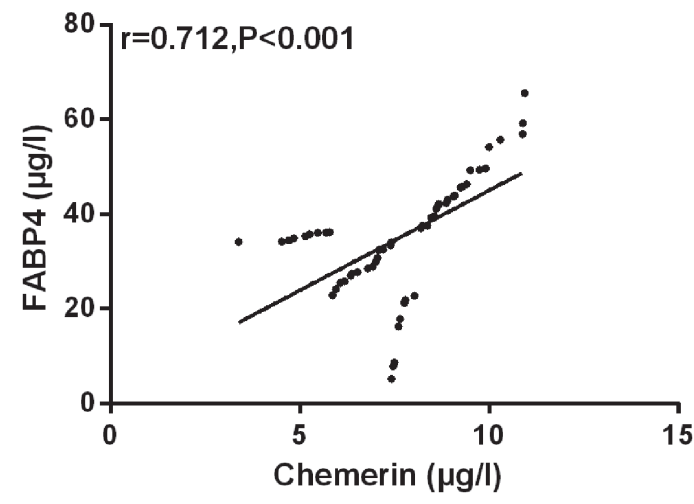

Figure 4. Chemerin is positively correlated with FABP4 $(r=0.712, \mathrm{P}<0.001)$. FABP4, fatty acid-binding protein 4.

indicating that Chemerin and FABP4 have certain diagnostic value for GDM and are important risk factors for GDM development. This is similar to the results of the present study. In the study of Zhang et al (26) on inflammation and metabolism of GDM patients in Inner Mongolia, the distribution frequency of inflammatory factors IL- 6 and TNF- $\alpha$ in placenta in GDM women was significantly higher than that in healthy pregnant women and IL-6 was significantly correlated with GDM disease, suggesting that overexpression of these two inflammatory mediators may aggravate the progression of GDM disease by activating inflammatory cascade reaction in placenta. Other studies have shown that elevated levels of IL- 6 and TNF- $\alpha$ in amniotic fluid of GDM patients may play an important role in the process of GDM (27). The results of this study on inflammatory factors showed that the expression of inflammatory factors IL-6 and TNF- $\alpha$ in SG was significantly higher than those in CG. Chemerin and FABP4 were significantly positively correlated with inflammatory factors IL-6 and TNF- $\alpha$, suggesting that the high expression of Chemerin and FABP4 in inflammatory environment may be related to the development and progression of GDM. In studies of Feng et al (28) on risk factors of GDM, advanced age, hepatitis B virus, family history of diabetes, high BMI before pregnancy and a large increase of weight before 24 weeks of pregnancy may all increase the risk of GDM. In this study, the results of Logistic multivariate regression analysis of affecting GDM showed that advanced age ( $\geq 35$ years), family history of diabetes, hyperlipidemia, high pre-pregnancy BMI, high fasting blood glucose, high Chemerin and high FABP4 expression are risk factors of GDM patients. Among them, high Chemerin and high FABP4 expression have the greatest risk multiple, indicating that knockdown of Chemerin and FABP4 expression may reduce the onset risk of GDM patients. In the report of Chung et al (29), a CRISPR system of interfering with FABP4 expression was directionally transmitted to white adipocytes, which showed improvement effect on obesity, inflammation and IR. In studies of Josephrajan et al (30) on the secretion mechanism of FABP4, the main secretion of FABP4 is the selective secretion mediated by autophagy, suggesting inhibition of the secretion of FABP4 by autophagy inhibitor to reduce the influence of FABP4 expression on IR in GDM patients. Chemerin and FABP4 in SG have significant positive correlation, which indicated that Chemerin and FABP4 may play a synergistic role in GDM, but the specific regulatory mechanism needs to be further determined by cytological function research.

This study confirmed the positive correlation between Chemerin and FABP4, both of which are overexpressed in GDM patients and have positive correlation with inflammatory factors IL-6 and TNF- $\alpha$.

In conclusion, Chemerin and FABP4 have satisfactory diagnostic value for GDM patients and inhibition of chemorin and FABP4 expression can be used as potential therapeutic targets for GDM patients.

\section{Acknowledgements}

Not applicable.

\section{Funding}

No funding was received.

\section{Availability of data and materials}

The datasets used and/or analyzed during the present study are available from the corresponding author on reasonable request. 


\section{Authors' contributions}

$\mathrm{XW}, \mathrm{JL}, \mathrm{DW}$ and JJ led the conception and design of this study. XW, JL, DW, HZ and LK were responsible for the data collection and analysis. XW, JL and JJ were in charge of interpreting the data and drafting the manuscript. HZ and LK made revision from critical perspective for important intellectual content. The final version was read and adopted by all the authors.

\section{Ethics approval and consent to participate}

The study was approved by the Ethics Committee of the People's Hospital of Zhangqiu Area (Jinan, China). Signed informed consents were obtained from the patients and/or guardians.

\section{Patient consent for publication}

Not applicable.

\section{Competing interests}

The authors declare that they have no competing interests.

\section{References}

1. Vokalova L, van Breda SV, Ye XL, Huhn EA, Than NG, Hasler P, Lapaire O, Hoesli I, Rossi SW and Hahn S: Excessive neutrophil activity in gestational diabetes mellitus: Could it contribute to the development of preeclampsia? Front Endocrinol (Lausanne) 9: $542,2018$.

2. Schaefer-Graf U, Napoli A and Nolan CJ; Diabetic Pregnancy Study Group: Diabetes in pregnancy: A new decade of challenges ahead. Diabetologia 61: 1012-1021, 2018.

3. Damm P, Houshmand-Oeregaard A, Kelstrup L, Lauenborg J, Mathiesen ER and Clausen TD: Gestational diabetes mellitus and long-term consequences for mother and offspring: A view from Denmark. Diabetologia 59: 1396-1399, 2016.

4. Sweeting AN, Ross GP, Hyett J, Molyneaux L, Constantino M, Harding AJ and Wong J: Gestational diabetes mellitus in early pregnancy: Evidence for poor pregnancy outcomes despite treatment. Diabetes Care 39: 75-81, 2016.

5. Pantham P, Aye IL and Powell TL: Inflammation in maternal obesity and gestational diabetes mellitus. Placenta 36: 709-715, 2015.

6. Powe CE, Allard C, Battista MC, Doyon M, Bouchard L, Ecker JL, Perron P, Florez JC, Thadhani R and Hivert MF: Heterogeneous contribution of insulin sensitivity and secretion defects to gestational diabetes mellitus. Diabetes Care 39: 1052-1055, 2016.

7. Du J,Zhu YL and Gao XM: Expressions of inflammatory cytokines and fat factors in placentas of patients with gestational diabetes mellitus and their relationship with glucose and lipid metabolism. Hainan Yixueyuan Xuebao 22: 39-42, 2016 (In Chinese).

8. Mariani F and Roncucci L: Chemerin/chemR23 axis in inflammation onset and resolution. Inflamm Res 64: 85-95, 2015.

9. Kennedy AJ and Davenport AP: International union of basic and clinical pharmacology CIII: Chemerin receptors CMKLR1 (Chemerin1) and GPR1 (Chemerin2) nomenclature, pharmacology, and function. Pharmacol Rev 70: 174-196, 2018.

10. Haberl EM, Pohl R, Rein-Fischboeck L, Feder S, Eisinger K, Krautbauer S, Sinal CJ and Buechler C: Ex vivo analysis of serum chemerin activity in murine models of obesity. Cytokine 104: 42-45, 2018.

11. Cetin O, Kurdoglu Z, Kurdoglu M and Sahin HG: Chemerin level in pregnancies complicated by preeclampsia and its relation with disease severity and neonatal outcomes. J Obstet Gynaecol 37: 195-199, 2017.

12. Yang X, Quan X, Lan Y, Wei Q, Ye J, Yin X, Ji Z, Xing H and Yang Y: Serum chemerin level in women with PCOS and its relation with the risk of spontaneous abortion. Gynecol Endocrinol 34: 864-867, 2018.
13. Ademoglu E, Berberoglu Z, Dellal FD and Ariel KM: Higher levels of circulating chemerin in obese women with gestational diabetes mellitus. Acta Endocrinol (Bucur) 11: 1841-0987, 2015.

14. Yang X, Quan X, Lan Y, Ye J, Wei Q, Yin X, Fan F and Xing H: Serum chemerin level during the first trimester of pregnancy and the risk of gestational diabetes mellitus. Gynecol Endocrinol 33: 770-773, 2017.

15. Guaita-Esteruelas S, Gumà J, Masana L and Borràs J: The peritumoural adipose tissue microenvironment and cancer. The roles of fatty acid binding protein 4 and fatty acid binding protein 5 . Mol Cell Endocrinol 462: 107-118, 2018.

16. Hertzel AV, Xu H, Downey M, Kvalheim N and Bernlohr DA: Fatty acid binding protein 4/aP2-dependent BLT1R expression and signaling. J Lipid Res 58: 1354-1361, 2017.

17. Ning $H$, Tao $H$, Weng $Z$ and Zhao X: Plasma fatty acid-binding protein 4 (FABP4) as a novel biomarker to predict gestational diabetes mellitus. Acta Diabetol 53: 891-898, 2016.

18. de Gennaro G, Palla G, Battini L, Simoncini T, Del Prato S, Bertolotto A and Bianchi C: The role of adipokines in the pathogenesis of gestational diabetes mellitus. Gynecol Endocrinol 35: 737-751, 2019.

19. American Diabetes Association: Classification and diagnosis of diabetes. Diabetes Care 40 (Suppl 1): S11-S24, 2017.

20. Chang L, Rissin DM, Fournier DR, Piech T, Patel PP, Wilson DH and Duffy DC: Single molecule enzyme-linked immunosorbent assays: theoretical considerations. J Immunol Methods 378: 102-115, 2012.

21. Chen Z, Gong L, Zhang P, Li Y, Liu B, Zhang L, Zhuang J and Xiao D: Epigenetic down-regulation of Sirt 1 via DNA methylation and oxidative stress signaling contributes to the gestational diabetes mellitus-induced fetal programming of heart ischemia-sensitive phenotype in late life. Int J Biol Sci 15: 1240-1251, 2019.

22. Muller PS and Nirmala M: Effects of pre pregnancy maternal body mass index on gestational diabetes mellitus. IACSIT Int J Eng Technol 7: 279-282, 2018.

23. Gilbert L, Gross J, Lanzi S, Quansah DY, Puder J and Horsch A: How diet, physical activity and psychosocial well-being interact in women with gestational diabetes mellitus: An integrative review. BMC Pregnancy Childbirth 19: 60, 2019.

24. Artal R, Catanzaro RB, Gavard JA, Mostello DJ and Friganza JC: A lifestyle intervention of weight-gain restriction: diet and exercise in obese women with gestational diabetes mellitus. Appl Physiol Nutr Metab 32: 596-601,2007.

25. Roca-Rodríguez MM, López-Tinoco C, Fernández-Deudero A, Murri M, García-Palacios MV, García-Valero MA, TinahonesMadueño FJ and Aguilar-Diosdado M: Adipokines and metabolic syndrome risk factors in women with previous gestational diabetes mellitus. Diabetes Metab Res Rev 28: 542-548, 2012.

26. Zhang J, Chi H, Xiao H, Tian X, Wang Y, Yun X and Xu Y: Interleukin-6 (IL-6) and tumor necrosis factor- $\alpha$ (TNF- $\alpha$ ) single nucleotide polymorphisms (SNPs), inflammation and metabolism in gestational diabetes mellitus in Inner Mongolia. Med Sci Monit 23: 4149-4157, 2017.

27. Melekoglu R, Ciftci O, Celik E, Yilmaz E and Bastemur AG: Evaluation of second trimester amniotic fluid ADAMTS4, ADAMTS5, interleukin- 6 and tumor necrosis factor- $\alpha$ levels in patients with gestational diabetes mellitus. J Obstet Gynaecol Res 45: 824-829, 2019.

28. Feng Y, Jiang CD, Chang AM, Shi Y, Gao J, Zhu L and Zhang Z: Interactions among insulin resistance, inflammation factors, obesity-related gene polymorphisms, environmental risk factors, and diet in the development of gestational diabetes mellitus. J Matern Fetal Neonatal Med 32: 339-347, 2019.

29. Chung JY, Ain QU, Song Y, Yong SB and Kim YH: Targeted delivery of CRISPR interference system against Fabp4 to white adipocytes ameliorates obesity, inflammation, hepatic steatosis, and insulin resistance. Genome Res 29: 1442-1452, 2019.

30. Josephrajan A, Hertzel AV, Bohm EK, McBurney MW, Imai SI, Mashek DG, Kim DH and Bernlohr DA: Unconventional secretion of adipocyte fatty acid binding protein (FABP4) by adipocytes. FASEB J 32: 814.11-814.11, 2018.

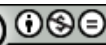

This work is licensed under a Creative Commons Attribution-NonCommercial-NoDerivatives 4.0 International (CC BY-NC-ND 4.0) License. 